\title{
Numerical Integration Formulas of Degree 3 for Product Regions and Cones
}

\author{
By A. H. Stroud
}

1. Introduction. Here we discuss approximate integration formulas of degree 3; that is, formulas which are exact for polynomials of degree $\leqq 3$, of the form

$$
\int_{R} f\left(x_{1}, \cdots, x_{n}\right) d x_{n} \cdots d x_{1} \simeq \sum b_{i} f\left(\nu_{i 1}, \cdots, \nu_{i n}\right)
$$

for certain regions $R$ in $n$-dimensional, real, Euclidean space $E_{n}$.

If $R$ is the Cartesian product of an $r$-dimensional and an $s$-dimensional region, $R=R_{r} \times R_{s}, r+s=n$, and if formulas of degree $k$ involving $p$ and $q$ points are known for $R_{r}$ and $R_{s}$, then Hammer and Wymore [4] have shown that a formula of degree $k$ may be obtained for $R$ which involves $p q$ points. Similarly, if $R$ is a cone (or pyramid) with $(n-1)$-dimensional base $B$, and if a formula of degree $k$ involving $p$ points is known for $B$, and further, if a formula of degree $k$ of the form

$$
\int_{0}^{1} x^{n-1} g(x) d x \simeq \sum a_{i} g\left(\nu_{i}\right)
$$

is known involving $q$ points, then Hammer, Marlowe and Stroud [2] have shown that a formula of degree $k$ can be obtained for $R$ which involves $p q$ points.

For $k=3$ we will show that these results can be improved as follows. If $R=$ $R_{r} \times R_{z}$ and formulas of degree 3 involving $p$ and $q$ points are known for $R_{r}$ and $R_{s}$, then a formula of degree 3 can be found for $R$ involving $p+q+1$ points (and in some cases this may be reduced to $p+q$ or $p+q-1$ points). If $R$ is a cone and a formula of degree 3 involving $p$ points is known for its base $B$, then a formula of degree 3 involving $p+3$ points (in some cases $p+2$ or $p+1$ points) can be found for $R$, whereas the method of [2] would involve $2 p$ points. We also give a $p+2$ point formula of degree 3 for certain double cones, where $p$ is the number of points in a formula for the base, and a $2 n+3$ point formula of degree 3 for an $n$-dimensional simplex.

2. Formulas for Product Regions. For convenience let us assume that the centroid of $R_{r}$ is at the origin of coordinates in the subspace $E_{r}$ of $E_{n}$ for which $x_{r+1}=\cdots=x_{n}=0$, and also that the centroid of $R_{z}$ is at the origin in the subspace $E_{s}$ for which $x_{1}=\cdots=x_{r}=0$. Then the centroid of $R$ is at the origin in $E_{n}$. Also, for convenience in notation, we will write

$$
R_{r}\left(x_{1}^{\alpha_{1}} \cdots x_{r}^{\alpha_{r}}\right) \equiv \int_{R_{r}} x_{1}^{\alpha_{1}} \cdots x_{r}^{\alpha_{r}} d x_{r} \cdots d x_{1}
$$

with similar notations for $R_{s}$ and $R$. Then, for example, $R_{r}(1)$ is the $r$-dimensional content or volume of $R_{r}$ and $R(1)=R_{r}(1) R_{s}(1)$ is the $n$-dimensional volume of $R$.

Received January 18, 1960. This paper was supported by the Graduate Research Committee of the University of Wisconsin. 
Suppose an approximate integration formula of degree 3 for $R_{r}$ is given by:

$$
\nu_{i}=\left(\nu_{i 1}, \cdots, \nu_{i r}\right) \quad a_{i}, \quad i=1,2, \cdots, p .
$$

Also suppose that a formula of degree 3 for $R_{s}$ is given by:

$$
\nu_{p+j}=\left(\nu_{p+j, r+1}, \cdots, \nu_{p+j, n}\right) \quad a_{p+j}, \quad j=1,2, \cdots, q .
$$

At first it is assumed no $\nu_{i}$ coincides with the origin in $E_{r}$ and no $\nu_{p+j}$ coincides with the origin in $E_{3}$. An integration formula for degree 3 for $R$ is given by:

$$
\begin{array}{rlrl}
\nu_{0}=(0, \cdots, 0,0, \cdots, 0) & b_{0}=-R(1) \\
\nu_{i}=\left(\nu_{i 1}, \cdots, \nu_{i r}, 0, \cdots, 0\right) & b_{i}=a_{i} R_{z}(1), \\
\nu_{p+j}=\left(0, \cdots, 0, \nu_{p+j, r+1}, \cdots, \nu_{p+j, n}\right) & i=1,2, \cdots, p \\
& b_{p+j}=a_{p+j} R_{r}(1), \\
j=1,2, \cdots, q .
\end{array}
$$

To prove this statement, it suffices to prove that the formula is exact for the following ten types of monomials: $1, x_{r_{1}}, x_{s_{1}}, x_{r_{1}} x_{r_{2}}, x_{r_{1}} x_{s_{1}}, x_{s_{1}} x_{s_{2}}, x_{r_{1}} x_{r_{2}} x_{r_{3}}, x_{r_{1}} x_{r_{2}} x_{s_{1}}, x_{r_{1}} x_{s_{1}}$ $x_{s_{2}}, x_{s_{1}} x_{s_{2}} x_{s_{3}}$ where $r_{1}, r_{2}, r_{3}=1,2, \cdots, r$ and $s_{1}, s_{2}, s_{3}=r+1, r+2, \cdots, n$ :

The monomial 1. Since $a_{1}+\cdots+a_{p}=R_{r}(1)$ and $a_{p+1}+\cdots+a_{p+q}=R_{s}(1)$ we have

$$
\begin{aligned}
-R(1)+\left[a_{1}+\cdots+a_{p}\right] R_{s}(1)+\left[a_{p+1}+\cdots\right. & \left.+a_{p+q}\right] R_{r}(1) \\
& =-R(1)+2 R_{r}(1) R_{s}(1)=R(1)
\end{aligned}
$$

The type $x_{r_{1}} x_{r_{2}} x_{r_{3}}$. The formula gives for the integral of $x_{r_{1}} x_{r_{2}} x_{r_{3}}$ over $R$

$$
\left[a_{1} \nu_{1 r_{2}} \nu_{1 r_{2}} \nu_{1 r_{3}}+\cdots+a_{p} \nu_{p r_{1}} \nu_{p r_{2}} \nu_{p r_{2}}\right] R_{s}(1)=R_{r}\left(x_{r_{1}} x_{r_{2}} x_{r_{3}}\right) R_{s}(1) .
$$

Thus the formula is exact because

$$
R\left(x_{r_{1}} x_{r_{2}} x_{r_{3}}\right)=R_{r}\left(x_{r_{1}} x_{r_{2}} x_{r_{3}}\right) R_{s}(1) .
$$

The proofs. for types $x_{r_{1}}, x_{s_{1}}, x_{r_{1}} x_{r_{2}}, x_{z_{1}} x_{s_{2}}, x_{z_{1}} x_{s_{2}} x_{s_{2}}$ are similar.

The type $x_{r_{1}} x_{r_{2}} x_{s_{1}}$. By the formula the integral of $x_{r_{1}} x_{r_{2}} x_{r_{1}}$ over $R$ is zero since each term of the sum is zero. However

$$
R\left(x_{r_{1}} x_{r_{2}} x_{s_{1}}\right)=R_{r}\left(x_{r_{1}} x_{r_{2}}\right) R_{s}\left(x_{s_{1}}\right)=0
$$

because $R_{s}\left(x_{s_{1}}\right)=0$. The proofs for the types $x_{r_{1}} x_{s_{1}}$ and $x_{r_{1}} x_{s_{1}} x_{s_{2}}$ are similar.

If the formulas for $R_{r}$ and $R_{s}$ already include the origin, then the formula for $R$ will involve either $p+q$ or $p+q-1$ points according as the origin is included in one or both of these formulas. In the former case, if $\nu_{p+1}$ in (2) is the origin, then $b_{0}=-\left[a_{p+2}+\cdots+a_{p+q}\right] R_{r}(1)$. In the latter case, if both $\nu_{1}$ in (1) and $v_{p+1}$ in (2) are the origin, then

$$
b_{0}=R(1)-\left[a_{2}+\cdots+a_{p}\right] R_{s}(1)-\left[a_{p+2}+\cdots+a_{p+4}\right] R_{r}(1) .
$$

Depending on the particular structure of the formulas (1) and (2) it may be possible in special cases to eliminate the origin in the formula (3) for $R$. Suppose in the formula (2) for $R_{s}, \nu_{p+1}$ is the origin and $a_{p+1}$ is positive. Also suppose $R_{r}$ is centrally symmetric. Then, as shown in [6], we may obtain $2 r$ point formulas for 
$R_{r}$ with $-v_{r+i}=\nu_{i}, a_{r+i}=a_{i}, i=1,2, \cdots, r$. In addition to the assumptions already made about $R_{r}$, we may also assume $R_{r}\left(x_{1}{ }^{2}\right)=\cdots=R_{r}\left(x_{r}{ }^{2}\right)$ and $R_{r}\left(x_{i} x_{j}\right)=$ 0 , for $i \neq j$; then in order that (1) be a formula of degree 3 it is necessary and sufficient that $\nu_{1}, \cdots, \nu_{r}$ be orthogonal vectors with $a_{i}=\frac{R_{r}\left(x_{1}{ }^{2}\right)}{2\left|\nu_{i}\right|^{2}}$ where $\left|\nu_{i}\right|$ is the distance of $\nu_{i}$ from the origin. Since in the formula for $R_{r} \times R_{d}$ it is no longer necessary that $b_{1}+\cdots+b_{2 r}=R(1)$, a formula of degree 3 for $R_{r} \times R_{s}$ is given by the $2 r+q-1$ points:

$$
\begin{array}{rc}
-\nu_{r+i}=\nu_{i}=\left(\nu_{i 1}, \cdots, \nu_{i r}, 0, \cdots, 0\right) & b_{r+i}=b_{i}, \\
\nu_{2 r+j}=\left(0, \cdots, 0, \nu_{2 r+j, r+1}, \cdots, \nu_{2 r+j, n}\right) & b_{2 r+j}=a_{2 r+j} R_{r}(1), \\
j=2, \cdots, q
\end{array}
$$

where the only conditions that the $b_{i}$ and $v_{i}, i=1,2, \cdots, r$, must satisfy are $2\left(b_{1}+\cdots+b_{r}\right)=a_{2 r+1} R_{r}(1)$ and $\nu_{1}, \cdots, \nu_{r}$ are any set of orthogonal vectors for which $\left|\nu_{i}\right|^{2}=\frac{R_{r}\left(x_{1}^{2}\right)}{2 b_{i}}$; the $b_{i}$ must all be positive.

Of course if both $R_{r}$ and $R_{s}$ are centrally symmetric then $R_{r} \times R_{s}$ is also centrally symmetric and the results of [6] may be applied directly to obtain $2 n$ point formulas. Some of the resulting formulas may also be obtained from the separate formulas for $R_{r}$ and $R_{s}$ by a method somewhat similar to that described in the preceding paragraph.

As an examplè of a specific formula we give a formula for the region $C_{r} \times S_{n-r}$, $1 \leqq r \leqq n-2$, where $C_{r}$ is the $r$-cube with vertices $( \pm 1, \pm 1, \cdots, \pm 1)$ and $S_{n-r}$ is the $(n-r)$-simplex with vertices $(-1,-1, \cdots,-1),(1,0, \cdots, 0)$, $(0,1, \cdots, 0), \cdots,(0,0, \cdots, 1)$. For $r=1, C_{1} \times S_{n-1}$ can be considered as a prism (or in the terminology of Sommerville [5], p. 113, a prism of the first species) with base $S_{n-1}$; for general $r, C_{r} \times S_{n-r}$ is a prism of species $r$. Since $C_{r}$ is centrally symmetric the remarks made above apply. A formula of degree 3 is known for $S_{n-r}$ using $n-r+2$ points, [3], one of which is the origin, but the origin is taken with a negative weight so that it is necessary to use $n+r+2$ points in the formula for $C_{r} \times S_{n \rightarrow r}$. A particular formula is:

$$
\begin{array}{cc}
\nu_{0}=(0,0, \cdots, 0,0,0, \cdots, 0) & b_{0}= \\
=2^{r} S_{n-r}(1)\left[1-\frac{r}{3}-\frac{(n-r+3)^{2}}{4(n-r+2)}\right] & b_{r+1}=b_{1}=\cdots= \\
-\nu_{r+1}=\nu_{1}=(1,0, \cdots, 0,0,0, \cdots, 0) & =b_{2 r}=b_{r}=\frac{2^{r-1}}{3} S_{n-r}(1) \\
-\nu_{r+2}=\nu_{2}=(0,1, \cdots, 0,00, \cdots, 0) & \\
-\nu_{2 r}=\nu_{r}=(0,0, \cdots, 1,0,0, \cdots, 0) & \\
\nu_{2 r+1}=\left(0,0, \cdots, 0, \frac{-2}{n-r+3},\right.
\end{array}
$$




$$
\begin{gathered}
\left.\quad \frac{-2}{n-r+3}, \cdots, \frac{-2}{n-r+3}\right) \quad b_{2 r+1}=\cdots=b_{n+r+1}= \\
\nu_{2 r+2}=\left(0,0, \cdots, 0, \frac{2}{n-r+3}, 0, \cdots, 0\right)=\frac{(n-r+3)^{2} 2^{r} S_{n-r}(1)}{4(n-r+1)(n-r+2)} \\
\cdots \\
\nu_{n+r+1}=\left(0,0, \cdots, 0,0,0, \cdots, \frac{2}{n-r+3}\right)
\end{gathered}
$$

3. Formulas for Cones. Suppose the centroid of the $(n-1)$-dimensional base $B$ is at the origin in the subspace $E_{n-1}$ of $E_{n}$ for which $x_{1}=0$ and that the vertex of $R$ is at $(1,0, \cdots, 0)$ in $E_{n}$. Suppose further a formula of degree 3 for $B$ is given by

$$
\nu_{i}=\left(\nu_{i 2}, \nu_{i 3}, \cdots, \nu_{i n}\right) \quad a_{i}, \quad i=1,2, \cdots, p .
$$

We assume at first that no $\nu_{i}$ coincides with the origin in $E_{n-1}$. An integration formula of degree 3 for $R$ can be found of the form

$$
\begin{aligned}
& \nu_{i}=\left(\eta, \gamma \nu_{i 2}, \gamma \nu_{i 3}, \cdots, \gamma \nu_{i n}\right) \quad b_{i}=\alpha a_{i}, i=1,2, \cdots, p \\
& \nu_{p+j}=\left(\xi_{j}, 0,0, \cdots, 0\right) \quad b_{j}, \quad j=1,2,3 .
\end{aligned}
$$

To prove this we must first calculate the monomial integrals over $R$ in terms of those over $B$. These are

$$
\begin{aligned}
\int_{R} x_{1}^{\beta} x_{2}^{\beta_{2}} \cdots x_{n}^{\beta_{n}} d x_{n} & \cdots d x_{1} \\
& =\int_{0}^{1}\left(1-x_{1}\right)^{n+\beta_{2}+\cdots+\beta_{n}-1} x_{1}^{\beta} d x_{1} \int_{B} x_{2}^{\beta_{2}} \cdots x_{n}^{\beta_{n}} d x_{n} \cdots d x_{2} .
\end{aligned}
$$

The equations which must be satisfied by (5) if it is to integrate each monomial are given below (where the monomial from which the equation arises is indicated at the left):

$$
\begin{aligned}
b_{1} \xi_{1}^{\beta}+b_{2} \xi_{2}^{\beta}+b_{3} \xi_{3}^{\beta}+\alpha \eta^{\beta}\left[a_{1}+\cdots+a_{p}\right]=\frac{\beta !(n-1) !}{(n+\beta) !} & B(1), \\
\beta & =0,1,2,3 .
\end{aligned}
$$

$\left[x_{1}^{\beta} x_{i}\right]$

$$
\alpha \gamma \eta^{\beta}\left[a_{1} \nu_{1 i}+\cdots+a_{p} \nu_{p i}\right]=\frac{\beta ! n !}{(n+\beta+1) !} B\left(x_{i}\right)
$$$$
\beta=0,1,2
$$

$\left[x_{1}^{\beta} x_{i} x_{j}\right]$

$$
\begin{array}{r}
\alpha \gamma^{2} \eta^{\beta}\left[a_{1} \nu_{1 i} \nu_{1 j}+\cdots+a_{p} \nu_{p i} \nu_{p j}\right]=\frac{\beta !(n+1) !}{(n+\beta+2) !} B\left(x_{i} x_{j}\right), \\
\beta=0,1
\end{array}
$$

$\left[x_{i} x_{j} x_{k}\right]$

$$
\alpha \gamma^{3}\left[a_{1} \nu_{1 i} \nu_{1 j} \nu_{1 k}+\cdots+a_{p} \nu_{p i} \nu_{p j} \nu_{p k}\right]=\frac{1}{n+3} B\left(x_{i} x_{j} x_{k}\right)
$$

where $i, j, k=2,3, \cdots, n$. Because we have assumed $B\left(x_{i}\right)=0$, the three equations $\left[x_{1}^{\beta} x_{i}\right]$ are of the form $0=0$. From the equations $\left[x_{i} x_{j}\right]$ and $\left[x_{1} x_{i} x_{j}\right]$ it imme- 
diately results that $\eta=1 /(n+3)$. From equations $\left[x_{i} x_{j}\right]$ and $\left[x_{i} x_{j} x_{k}\right]$ it follows that

$$
\alpha=\frac{(n+3)^{2}}{(n+2)^{2}}, \quad \gamma=\frac{n+2}{n+3} .
$$

Since $a_{1}+\cdots+a_{p}=B(1)$ the equations that remain become

$$
\begin{aligned}
{\left[x_{1}^{\beta}\right] \quad b_{1} \xi_{1}^{\beta}+b_{2} \xi_{2}^{\beta}+b_{3} \xi_{2}^{\beta}+\frac{(n+3)^{2}}{(n+2)^{3}} \frac{1}{(n+3)^{\beta}} B(1)=\frac{\beta !(n-1) !}{(n+\beta) !} B(1), } \\
\beta=0,1,2,3 .
\end{aligned}
$$

In these equations we cannot take, say, $b_{3}=0$, which would in effect reduce by one the number of points in the formula, because the resulting equations do not have a real solution. It might be expected, however, that there would be many solutions with all $b_{i} \neq 0$. Here we give just one of the simplest solutions. If we choose

$$
b_{3}=-\frac{(n+3)^{2}}{(n+2)^{3}} B(1), \quad \xi_{3}=\frac{1}{n+3}
$$

then the other values are

$$
\begin{gathered}
b_{1}=b_{1}{ }^{\prime} B(1)=\frac{2(n+1)-(n-1) \sqrt{2(n+1)(n+2)}}{4 n(n+1)^{2}} B(1) \\
\xi_{1}=\frac{2(n+2)+\sqrt{2(n+1)(n+2)}}{(n+2)(n+3)} \\
b_{2}=b_{2}{ }^{\prime} B(1)=\frac{2(n+1)+(n-1) \sqrt{2(n+1)(n+2)}}{4 n(n+1)^{2}} B(1) \\
\xi_{2}=\frac{2(n+2)-\sqrt{2(n+1)(n+2)}}{(n+2)(n+3)} .
\end{gathered}
$$

These values of $b_{1}{ }^{\prime}, b_{2}{ }^{\prime}, \xi_{1}, \xi_{2}$ have been given in connection with numerical integration with respect to a weight function $x^{n-1}$ by Fishman [1] for $n=1(1) 6$ to $12 \mathrm{D}$ and in [2] for $n=2(1) 4$ to $18 \mathrm{~S}$. These authors have used $n$ where we use $n-1$ and for $m=2, j=1,2$, their $1-x_{j}$ is our $\xi_{j}$, their $b_{j}$ is our $b_{j}{ }^{\prime}$. It is proved in [2] that the $2 p$ points

$$
\left(\xi_{j},\left(1-\xi_{j}\right) \nu_{i 2},\left(1-\xi_{j}\right) \nu_{i 3}, \cdots,\left(1-\xi_{j}\right) \nu_{i n}\right) \quad b,^{\prime} a_{i}
$$

$i=1,2, \cdots, p, j=1,2$, are an integration formula of degree 3 for $R$.

If one of the $\nu_{i}$ in (4), say $\nu_{1}$, is the origin in $E_{n-1}$ then it may be possible to determine $b_{1}, b_{2}, \xi_{1}, \xi_{2}$ with $b_{2}=0$ to give a formula which involves only $p+1$ points $\nu_{2}, \cdots, \nu_{p+2}$. The formula using $n+2$ points when $R$ is an $n$-simplex, [3], can be derived in this manner; the formula for $B$, which is an $(n-1)$-simplex, involves $n+1$ points of which one is the origin in $E_{n-1}$. In any event, if $v_{1}$ is the origin, we may derive a formula of degree 3 for $R$ involving $p+2$ points $\nu_{2}, \cdots$, $\nu_{p+3}$ where

$$
b_{s}=-\frac{(n+3)^{2}}{(n+2)^{3}}\left[a_{z}+\cdots+a_{p}\right]
$$

and the other values as before. Usually there will be other $p+2$ point formulas as well. 
Now we briefly discuss formulas for regions which are double cones; that is, regions which are the union of two cones with vertices $(1,0, \cdots, 0)$ and $(-1,0, \cdots, 0)$ and with a common base $B$ of the type we have just considered. By a combination of the methods of this section and the preceding it is easy to see that from a formula (4) for $B$ we may obtain the following formula involving $p+2$ points for the double cone $R$ :

$$
\begin{array}{rlrl}
\nu_{i} & =\left(0, \gamma \nu_{i 2}, \gamma \nu_{i z}, \cdots, \gamma \nu_{i n}\right) & b_{i} & =\frac{2(n+3)^{2}}{(n+2)^{3}} a_{i}, \quad i=1,2, \cdots, p \\
-\nu_{p+2} & =\nu_{p+1}=(\xi, 0,0, \cdots, 0) \quad b_{p+2}=b_{p+1}=\frac{3 n+8}{n(n+2)^{3}} B(1)
\end{array}
$$

where

$$
\gamma=\frac{n+2}{n+3} \text { and } \xi=\sqrt{\frac{2 n^{2}+8 n+8}{3 n^{2}+11 n+8}}
$$

Since $\xi<1$ for all $n$, the points $v_{p+1}$ and $v_{p+2}$ are always interior to $R$. In this formula the origin is not required if it does not occur in (4) and $b_{p+1}$ and $\xi$ are uniquely determined. If the origin does occur in (4), then in some cases it can be eliminated in the formula for $R$; if $v_{1}$ is the origin, then $b_{p+1}, \xi$, and $b_{1}$ will not be uniquely determined and we may take $\xi$ arbitrary, which will then determine

$$
\begin{aligned}
b_{p+1} & =\frac{2}{\xi n(n+1)(n+2)} B(1) \\
b_{1} & =\frac{2}{n} B(1)-2 b_{p+1}-\frac{2(n+3)^{2}}{(n+2)^{2}}\left[a_{2}+\cdots+a_{p}\right] .
\end{aligned}
$$

Since $b_{p+1}$ is required to be positive, we could, providing

$$
\left[a_{2}+\cdots+a_{p}\right]<\frac{(n+2)^{2}}{n(n+3)^{2}} B(1),
$$

or, in other words, providing

$$
a_{1}>-\frac{(3 n+8)}{n(n+3)^{2}} B(1)
$$

choose $b_{1}=0$. In this case the formula for $R$ would involve only the $p+1$ points $\nu_{2}, \cdots, \nu_{p+2}$ and $b_{p+1}$ and $\xi$ would again be uniquely determined. In this paragraph $R$ has been a double cone of the first species with base $B$; this method may be repeated to give a formula for a double cone of species $r$. If we take a double cone of species $r$ with base $S_{n \rightarrow r}, 1 \leqq r \leqq n-2$, then this region has a formula of degree 3 which involves $n+r+2$ points.

4. A Special Formula for Simplexes. We digress here from the methods of the previous sections to give a special formula of degree 3 for a simplex $S_{n}$ in $E_{n}$. The formula involves $2 n+3$ points, $n \geqq 2$, all but one of which are on the surface of $S_{n}$; the formula involves the $n+1$ vertices, the $n+1$ centroids of the $(n-1)$ dimensional faces, and the centroid of $S_{n}$. 
To develop the formula it is most convenient to take $S_{n}$ to have vertices $(0,0, \cdots, 0),(1,0, \cdots, 0), \cdots,(0,0, \cdots, 1)$. Then the monomial integrals over $S_{n}$ are

$$
\int_{S_{n}} x_{i}^{\alpha_{i}} x_{j}^{\alpha_{j}} x_{k}^{\alpha_{k}} d x_{n} \cdots d x_{1}=\frac{\alpha_{i} ! \alpha_{j} ! \alpha_{k} !}{\left(n+\alpha_{i}+\alpha_{j}+\alpha_{k}\right) !} .
$$

The formula is then:

$$
\begin{array}{rlrl}
\nu_{0} & =\left(\frac{1}{n+1}, \frac{1}{n+1}, \cdots, \frac{1}{n+1}\right) & b_{0} & =-\frac{(n+1)^{2}(n-3)}{(n+2)(n+3)} S_{n}(1) \\
\nu_{1} & =(0,0, \cdots, 0) & b_{1} & =\cdots=b_{n+1}= \\
\nu_{2} & =(1,0, \cdots, 0) & & =\frac{3}{(n+1)(n+2)(n+3)} S_{n}(1) \\
& \cdots & & \\
\nu_{n+1} & =(0,0, \cdots, 1) & & =\cdots=b_{2 n+2}= \\
\nu_{n+2} & =\left(\frac{1}{n}, \frac{1}{n}, \cdots, \frac{1}{n}\right) \\
\nu_{n+3} & =\left(0, \frac{1}{n}, \cdots, \frac{1}{n}\right) & & \\
& & &
\end{array}
$$

where $S_{n}(1)=1 /(n !)$ is the volume of $S_{n}$. Because of the symmetries of this particular simplex, the proof that this is a formula of degree 3 can be established by verifying that it is exact for the 7 monomials: $1, x_{1}, x_{1}{ }^{2}, x_{1} x_{2}, x_{1}{ }^{3}, x_{1}{ }^{2} x_{2}, x_{1} x_{2} x_{3}$. For $n=1$ this formula gives us Simpson's formula, since for $S_{1}$, a line segment, the vertices coincide with the $(n-1)$-faces; the weight for each end point of $S_{1}$ is $b_{1}+b_{4}=b_{2}+b_{3}$. For $n=3, b_{0}=0$, and in this one case the centroid does not occur in the formula; for $n>3, b_{0}$ is negative.

This formula will be useful when it is desired to integrate over a region by subdividing the region into simplexes and then applying a formula of degree 3 to each simplex. For a large number of subdivisions the total number of points used in applying this formula to each simplex will be less than the total number of points used if the $n+2$ point formula is applied to each simplex. For example, for $n=3$ suppose we desire to integrate ovcr an icosahedron (which has 20 triangular faces) by subdividing it into 20 tetrahedra (each tetrahedron having as vertices the vertices of a face plus the center of the icosahedron). Repeated use of the formula given here would involve a total of 63 points whereas use of the 5 -point formula for each tetrahedron would involve 100 points.

5. Concluding Remarks. From the formulas we have discussed, formulas may be obtained for regions which are linear transforms of the particular regions we have cousidered. While these results add greatly to our knowledge of formulas of degree 3 
nothing is yet known concerning such formulas for regions which are less regular than those considered here.

It seems certain, although no proof is known, that the $n+2$ point formula of degree 3 for $S_{n}$ and the $2 n$ point formulas of degree 3 for $C_{n}$ involve the minimal number of points for this degree. If this is true, then it is also likely that the $\mathrm{n}+$ $r+2$ point formulas for both $C_{r} \times S_{n-r}$ and the double cone of species $r$ with base $S_{n-r}$ are also minimal. For fixed $r$ these latter regions are the duals of each other (see [5], p. 56) and thus have the same symmetries. This seems to indicate that the minimal point formulas of degree 3 for a region are related to the group of symmetries of the region.

I am indebted to R. G. Hetherington for many discussions on this subject.

Department of Economics

University of Wisconsin, Madison

1. H. Fishman, "Numerical integration constants," ${ }^{2}$ T A A ', v. 11, 1957, p. 1-9.

2. P. C. HAMNer, O. J. MARLOWE \& A. H. Stroud, "Numerical integration over simplexes and cones," MTAC, v. 10, 1956, p. 130-137.

3. P. C. HAMMer \& A. H. STroud, "Numerical integration over simplexes," IITAC, v. 10,1956, p. $137-139$.

4. P. C. HAMMer \& A. W. Wrmore, "Numerical evaluation of multiple integrals I," $M T A C$, v. 11, 1957, p. 59-67.

5. D. M. Y. Somarenville, An Introduction to the Geometry of $N$ Dimensions, Methuen, London, 1929.

6. A. H. Strocd, "Numerical integration formulas of degree two," Math. Comp., v. 14, 1960 , p. 21. 